\title{
Moist static energy: definition, reference constants, a conservation law, and effects on buoyancy
}

Article

Accepted Version

Yano, J.-I. and Ambaum, M. H. P. (2017) Moist static energy: definition, reference constants, a conservation law, and effects on buoyancy. Quarterly Journal of the Royal Meteorological Society, 143 (708). pp. 2727-2734. ISSN 1477-870X doi: https://doi.org/10.1002/qj.3121 Available at https://centaur.reading.ac.uk/71775/

It is advisable to refer to the publisher's version if you intend to cite from the work. See Guidance on citing.

Published version at: http://dx.doi.org/10.1002/qj.3121

To link to this article DOI: http://dx.doi.org/10.1002/qj.3121

Publisher: Royal Meteorological Society

All outputs in CentAUR are protected by Intellectual Property Rights law, including copyright law. Copyright and IPR is retained by the creators or other copyright holders. Terms and conditions for use of this material are defined in the End User Agreement.

www.reading.ac.uk/centaur 
Central Archive at the University of Reading

Reading's research outputs online 


\title{
Moist Static Energy : Definition, Reference Constants, a
}

\section{Conservation Law, and Effects on Buoyancy}

\author{
JUN-ICHI YANO ${ }^{\mathrm{a} *}$, MAARTEN H. P. AMBAUM ${ }^{\mathrm{b}}$ \\ ${ }^{a}$ CNRM (CNRS and Météo-France), 31057 Toulouse Cedex, France \\ ${ }^{\mathrm{b}}$ Department of Meteorology, University of Reading, UK
}

( ${ }^{*}$ Correspondence to: CNRM, Météo-France, 42 av Coriolis, 31057 Toulouse Cedex, France. E-mail: jiy.gfder@ gmail.com.

Atmospheric thermodynamic variables are commonly computed under approximations. Although exact formulas are available, they are rarely used. This paper addresses some potential issues arising when using approximate formulas by taking the moist static energy as an example. An important conclusion is that the temperature dependence of latent heat must be taken into account. We also demonstrate that the zeropoint energies of various species do not affect the moist static energy budget. The use of an exact formula for moist static energy increases its surface value by $15 \mathrm{~K}$ for a typical tropical sounding. However, the change of the parcel buoyancy by using the exact formula is less dramatic, although not negligible. Calculating, for example, the CAPE for convection parameterization, the use of an exact formula is likely not be critical for the practical purposes, but quantitative discrepancies can be as large as 50-200 J/kg.

Key Words: moist static energy; buoyancy; convective available potential energy; tropical atmosphere

Received...

(1)

1. Introduction

Thermodynamic variables are often presented in approximate forms. Studies exist presenting the definitions of these variables in exact forms (Hauf and Höller 1987, Ooyama 1990, Marquet 1993, 2011, 2015, Emanuel 1994, Murphy and Toop 2005; Marquet and Geleyn 2013). However, data analysis and modelling studies in the literature rarely use these exact definitions. The motivation of this study is to examine whether the exact definitions of thermodynamic variables have important consequences, or whether approximate relations may be sufficient.

We take the moist static energy ( $c f$. ., Marquet 1993, 2015, Emanuel 1994) as a specific example in this study. We address the following issues: first, we relate the exact definition of the moist static energy to the standard approximation (Sec. 2). Second, as it turns out, under a rigorous derivation, its definition contains reference constants, which can only be determined by laboratory measurements or full quantum-mechanical calculations. An obvious question to ask is the role of these constants in order to maintain a conservation law associated with the moist static energy (Sec. 3). Some further issues concerning these constants are also discussed in

This article has been accepted for publication and undergone full peer review but has not been through the copyediting, typesetting, pagination and proofreading process, which may lead to differences between this version and the Version of Record. Please cite this article as doi: 10.1002/qj.3121 
the Appendix A, being partially inspired by an analogous discussion on entropy by Pauluis et al. (2010). Third, perhaps surprisingly, a large discrepancy between the exact definition and the standard approximate definition of moist static energy is found, reaching about $15 \mathrm{~K}$ for typical tropical values. But how serious is this discrepancy? In order to answer this question, we evaluate the lifting-parcel buoyancy based on these two definitions by invoking the conservation of moist static energy under this process (Sec. 4). We also address the change of the values in CAPE (convective available potential energy) as a result.

\section{Definition}

Moist static energy is conserved under moist adiabatic processes and under hydrostatic balance, thus it is a useful quantity to understand oist convection. Under the standard approximation, its specific value (value per total air mass) is defined by

$$
h=c_{p d} T+q_{v} L+g z
$$

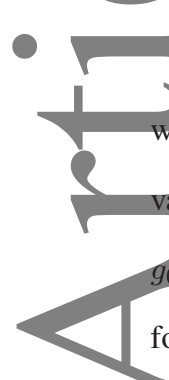

where $c_{p d}$ is the heat capacity of the dry air at constant pressure, $T$ the temperature, $q_{v}$ the specific vapour value, $L$ latent heat of vapourization of liquid water, $g$ acceleration of the gravity, $z$ the altitude. The geopotential contribution, $g z$, is an approximation of $Z$ with $g_{0}$ a reference value for the acceleration of gravity and $Z$ the geopotential height. The present approximation is very accurate for altitudes below about $50 \mathrm{~km}$, and its effect will therefore be ignored here.

The physical meaning of this definition of moist static energy is relatively intuitive: the first term is the dry-air enthalpy (or "heat content"), the second term is a potential contribution to the first term due to latent heating, and the last term is the specific gravitational potential energy. It is also intuitively expected that the sum of these three terms would be conserved under adiabatic and hydrostatic transformations. Hydrostatic balance ensures that any change in gravitational potential energy is compensated for by a change of enthalpy through a change in pressure.

(1)

In deriving an exact expression, we may divide the above expression into the two contributions: the "proper" enthalpy (the first two terms), $\tilde{h}$, and the gravitational potential energy. Thus,

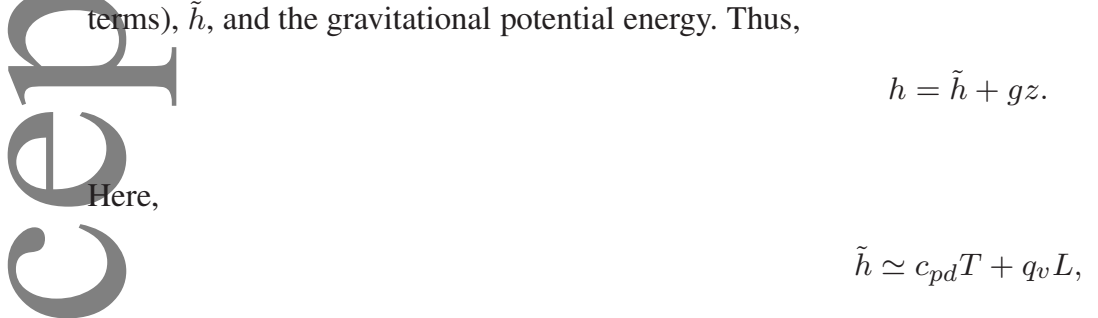

which corresponds to a standard definition of enthalpy found in textbooks on thermodynamics, which are mostly concerned with laboratory-scale processes, where changes in gravitational potential can be neglected. When changes in potential energy are important, the quantity $h$ becomes the conserved variable. Ambaum (2010, Sec. 5.4) calls $h$ the generalized enthalpy. Under standard approximations (namely the ideal gas law for any gaseous components and incompressibility assumptions for the solid and liquid components), the generalized enthalpy reduces to what is normally called the moist static energy. Here, we will adopt this standard terminology, and call $\tilde{h}$ the enthalpy, and $h$ the moist static energy.

Taking the ideal gas approximation, the specific enthalpy is given by a sum of contributions from dry air, $h_{d}$, water vapour, $h_{v}$, and the liquid water, $h_{l}$. Thus,

$$
\tilde{h}=\left(1-q_{t}\right) h_{d}+q_{v} h_{v}+q_{l} h_{l}
$$

where $q_{l}$ is the specific liquid water, and $q_{t}=q_{v}+q_{l}$. A contribution of ice is considered separately in Appendix B for keeping the mathematical expressions in main text as simple as possible.

This article is protected by copyright. All rights reserved. 
Each specific enthalpy value is defined by

$$
h_{d}=h_{0 d}+c_{p d} T, \quad h_{v}=h_{0 v}+c_{p v} T, \quad h_{l}=h_{0 l}+c_{l} T
$$

in which the first term is a reference constant (a zero-point energy), and the second term is a relative value proportional to the specific heat at constant pressure for each component. This simple form for the gaseous components assumes that they are ideal gases, an assumption which is very accurate for atmospheric temperature and pressure ranges. The simple form for the liquid component assumes a constant specific heat capacity as well as incompressibility. Again, these assumptions are accurate for atmospheric temperature and pressure ranges down to $-20^{\circ} \mathrm{C}(c f$., Fig. 6 of Murphy and Koop 2005). The effect of the temperature dependence in the liquid-water heat capacity is examined separately in Appendix C.

Strictly speaking, each expression in Eq. (5) must be written in the form $(1)$ with

$$
h_{j}=h_{0 j}+\int_{0}^{T} c_{p j}\left(T^{\prime}\right) d T^{\prime}
$$

with the subscript $j$ suggesting a component with the heat capacity, $c_{p j}$, at constant pressure defined as a function of temperature. Uhder this strict expression, the reference value, $h_{0 j}$, has a clear physical meaning as an enthalpy value extrapolated to zero absolute temperature, excluding phase transitions ( $c f .$, Kittel and Kroemer 1980, Ch. 6). However, the use of this strict expression for practical computations is difficult especially considering the large uncertainties in data towards the limit of the absolute zero temperature, as reviewed by Murphy and Koop (2005). For this reason, we take Eq. (5), that is considered a good approximation for this rigorous expression around a temperature range of interest.

$(1)$

The specific enthalpies for vapour and liquid water are related to the latent heat of vapourization, $L$, by

$$
L=h_{v}-h_{l} .
$$

Removing $h_{v}$ by Eq. (6) in Eq. (4), and substituting the remaining expressions from Eq. (5), we obtain

$$
\tilde{h}=\tilde{c}_{p} T+q_{v} L+\left(1-q_{t}\right) h_{0 d}+q_{t} h_{0 l},
$$

$$
\tilde{c}_{p}=\left(1-q_{t}\right) c_{p d}+q_{t} c_{l}
$$

Eq. (7) may be considered the final expression sought, as presented in e.g., Marquet (1993, 2015), Emanuel (1994). An alternative form called "liquid-water" enthalpy (e.g., Emanuel 1994), obtained by removing $h_{l}$ instead, is discussed separately in Appendix A.

Equation (7) is, however, hard to interpret in an intuitive manner: we expect that the heat capacity, $c_{p d}$, for the dry air in Eq. (3) would be replaced by that for the total air (including contained condensed water), which is equal to

$$
c_{p}=\left(1-q_{t}\right) c_{p d}+q_{v} c_{p v}+q_{l} c_{l} .
$$

This article is protected by copyright. All rights reserved. 
However, instead of $c_{p}$, we get $\tilde{c}_{p}$ defined by Eq. (8). It is also not obvious why the total water is weighted by the reference constant $h_{0 l}$, for liquid-water enthalpy in the last term. It might have been more intuitive to expect, $h_{0 v}$, especially when the air is unsaturated, thus $q_{t}=q_{v}$. Of course this counterintuitive outcome is the result of standard algebraic manipulation. However, its form is not unique.

This counterintuitive outcome may be alleviated when we notice that substitution of Eq. (5) into Eq. (6) leads to an integral version of Kirchhoff's equation for ideal gases (Ambaum, 2010, Sec. 3.6):

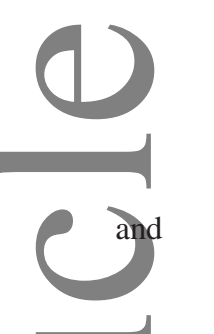

$$
\begin{gathered}
L=L_{0}-\left(c_{l}-c_{p v}\right) T, \\
L_{0}=h_{0 v}-h_{0 l},
\end{gathered}
$$

which can be seen as the linear extrapolation of the latent heat of evaporation to zero absolute temperature. For water vapour extrapolated from typical atmospheric temperatures, we find

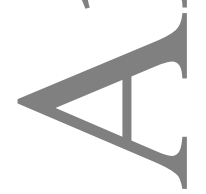

$$
L_{0}=3.14 \times 10^{6} \mathrm{~J} \mathrm{~kg}^{-1}
$$

Recall that the latent heat of evaporation for typical atmospheric temperatures is approximately

$$
L \simeq 2.5 \times 10^{6} \mathrm{~J} \mathrm{~kg}^{-1}
$$

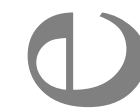

1

Substitution of Eq. (10) into Eq. (7) finally leads to:

$$
\tilde{h}=c_{p} T+q_{v} L_{0}+h_{0 d}+q_{t}\left(h_{0 l}-h_{0 d}\right)
$$

63 This expression (12) is much easier to interpret against the standard approximate expression (3): an exact expression (12) is obtained from an approximation (3) by replacing $c_{p d}$ by $c_{p}$ and $L$ by $L_{0}$. Furthermore, a reference constant value must be added. Although the alue of the reference constant may remain somewhat counterintuitive, it is now clear that its value is determined by splitting it out 66 into the dry-air and the water dependences on the reference constants. Note also that $L_{0}$ is about $25 \%$ larger than the typical expected value of latent heat, $L$, of evaporation. This will have a substantial effect on the calculated values of moist static energy, as is going to

68 beladdressed in Sec. 4.

It may be tempting to conclude that for achieving physically consistent expressions involving enthalpy, all we need to do is to replace the dry heat capacity, $c_{p d}$, by the full parcel heat capacity, $c_{p}$, and the latent heat, $L$, by its extrapolated value $L_{0}$. However, this is not generally the case. For example consider the effect of condensation on the temperature of a parcel. A standard procedure, as found in Asai (1965), Yau and Austin (1979), Rutledge and Hobbs (1983), Grabowski(1988, 1989), invokes the relation:

$$
-L \Delta q_{v}=c_{p} \Delta T
$$

This article is protected by copyright. All rights reserved. 
where $-\Delta q_{v}$ is the amount of condensation, and $\Delta T$ is the resulting increase of temperature by latent heating. Often, the heat capacity at constant pressure is approximated by that of the dry air, thus $c_{p} \simeq c_{p d}$. Here, this equation simply states that the amount of latent heating (left hand side) balances with the increase of the "heat content" of the air (right hand side).

The above formula appears to be based on the assumption that the enthalpy is defined by

$$
\tilde{h}=c_{p} T+q v L
$$

(1)

that enthalpy is conserved under the condensation process (Ambaum 2010, Sec. 5.4). If we replace this equation by the exact version Eq 12, then it does not follow that $-L_{0} \Delta q_{v}=c_{p} \Delta T$, because the changing composition following condensation also changes

the heat capacity. So the appropriate equation for the condensation is

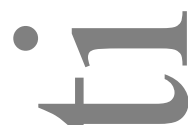

$$
-L_{0} \Delta q_{v}=c_{p} \Delta T+T \Delta c_{p}
$$

This equation turns out to be equivalent to Eq. (13), when the full heat capacity is used, $L$ is the usual temperature dependent latent helat as given by Eq. (10), and conservation of total water is invoked so that $\Delta q_{l}=-\Delta q_{v}$.

\section{Reference Constants and a Conservation Law}

The final result, Eq. (12) above, is fairly close to the standard approximate expression, Eq. (3), except for the two additional terms inyolving the reference constants. The first reference constant term, $h_{0 d}$, is just a constant, and it does not change by any processes. Thus, $h_{0 d}$ can take any arbitrary value, and we may set $h_{0 d}=0$ without loss of generality. On the other hand, the second reference constant term is proportional to the specific total water, $q_{t}$. Thus, the value of this term changes with $q_{t}$, and it looks like that the ehoice of the value for $h_{0 l}-h_{0 d}$ would affect the whole budget for the moist static energy when rain falls out of the parcel (e.g., when considering pseudo-adiabatic ascent). We now examine how the value of $h_{0 l}-h_{0 d}$ affects the budget more precisely. For this purpose, we take the total derivative of the moist static energy defined by Eq. (12):

$$
d \tilde{h}=c_{p} d T+T d c_{p}+L_{0} d q_{v}+\left(h_{0 l}-h_{0 d}\right) d q_{t}
$$

The heat capacity at constant pressure is not constant anymore, but

$$
d c_{p}=\left(c_{l}-c_{p d}\right) d q_{t}-\left(c_{l}-c_{p v}\right) d q_{v}
$$

By substituting Eq. (16) into Eq. (15), and after some rearrangements by recalling Eq. (10), we obtain

$$
d \tilde{h}=c_{p} d T+L d q_{v}+\left(h_{l}-h_{d}\right) d q_{t}
$$

This equation provides the rationale for E. (13), and the subsequent discussion. Note that the value of $h_{l}-h_{d}$ depends on the reference constants $h_{0 l}-h_{0 d}$. This is different from the dependence on reference constants of $L=h_{v}-h_{l}$; the latter is a measurable property of a given substance which only depends on the change in reference constants across the phase transition, not on the absolute value of the constants themselves, whilst $h_{0 l}-h_{0 d}$ is not directly measurable in classical experiments and is not a property of a given 
substance. It is an artificial property in the specific enthalpy budget resulting only from changing the mass ratio between the liquid and dry components.

Inspection of Eq. (17) leads to two conclusions:

(i) When the total water is conserved, and $d q_{t}=0$, the constant, $h_{0 l}-h_{0 d}$, does not affect the budget.

(ii) When the total water is not conserved, and $d q_{t} \neq 0$, the constant, $h_{0 l}-h_{0 d}$, must be specified. Especially, when the total water is lost by precipitation, the constant, $h_{0 l}-h_{0 d}$, defines the rate, $\left(h_{l}-h_{d}\right) d q_{t}$, that the enthalpy is lost locally by transport associated with precipitation. Thus, the enthalpy transport rate by precipitation depends on a choice of the value of $h_{0 l}-h_{0 d}$.

In order to close the enthalpy budget, the enthalpy transport rate must be specified. For this purpose, the value of $h_{0 l}-h_{0 d}$ must also specified. As an amount of total water, $d q_{t}$, is transported from one position to another, an amount of enthalpy, $\left(h_{0 l}-h_{0 d}\right) d q_{t}$, is also transported. However, as the above argument suggests, its actual value plays no other part in the thermodynamics of the parcel. It is needed only for book keeping the specific enthalpy, when a parcel composition changes through gravitational separation of the liquid (and solid) parts of the parcel. The consistency of the enthalpy budget is maintained regardless of the value assigned to $h_{0 l}-h_{0 d}$. Thus, $h_{0 l}-h_{0 d}$ can be specified in an arbitrary manner.

The reason for $h_{0 l}-h_{0 d}$ not playing any part in the thermodynamics of the parcel may further be understood in the following manner. The precipitation process (i.e., $d q_{t}=d q_{l}<0$ ) separates out the precipitable water from the rest of the parcel by taking the two steps: 1) the separation within the parcel of the precipitating liquid and the rest of the mixture in the parcel, and 2) the removal of the precipitating liquid from the parcel. In the first step, nothing happens to the total parcel, but the enthalpy in the parcel can be allocated separately to the precipitable water (an amount of $-h_{l} d q_{t}$ per unit total mass) and to the rest of the mixture. In the second step of the precipitation process, the liquid and its enthalpy is removed from the parcel mixture, but in this step the mass fraction of the dry air in the parcel mixture has increased by an amount $-d q_{t}$. Any first order effect of changing mass fraction of the vapour is captured in the second term of Eq. (17), but is in any case small because it is proportional to $d q_{t}$. The net change in specific enthalpy, therefore, must be $\left(h_{l}-h_{d}\right) d q_{t}$. Here, there is no thermodynamic transformation involved, just a recognition that different composition mixtures will have different values for the zero-point specific enthalpy values. When re-calculating the enthalpy of the new mixture, the same reference constants for the components can be used.

C Und

Under these considerations, we can set $h_{0 l}-h_{0 d}=0$ in the following analysis, so that the change of composition due to precipitation does not show up as an apparent change of reference constant of the mixture.

As a final word of warning (and unfortunate additional confusion), however, different choices for these constant values indeed lead to different values for the enthalpy. An equivalent issue with entropy is extensively discussed by Pauluis et al. (2010). The same is discussed in the Appendix A separately with a further discussion on the significance of those constant values.

\section{Data Analysis}

Our next goal is to examine the extent that any data analysis results are modified by using the exact definition (12) for the enthalpy instead of the standard approximate definition (3). For this purpose, we use two data sets. The first is a mean Caribbean sounding for July-October (for the hurricane season) assembled by Jordan (1958), and as given by his Table 5. This sounding provides values from the surface $(1015 \mathrm{hPa})$ to $30 \mathrm{hPa}$ level with a vertical resolution of $50 \mathrm{hPa}$ from $1000 \mathrm{hPa}$ to $200 \mathrm{hPa}, 25 \mathrm{hPa}$ above $200 \mathrm{hPa}, 20 \mathrm{hPa}$ above $100 \mathrm{hPa}$, and $10 \mathrm{hPa}$ above $60 \mathrm{hPa}$.

This article is protected by copyright. All rights reserved. 
The second data set is over the Intensive Flux Array (IFA) during the TOGA-COARE (Tropical Ocean Global Atmosphere Coupled Ocean Atmosphere Response Experiment) Intensive Observing Period (IOP — 1 November, 1992 through to 28 February, 1993). The data set is processed at the State University of Colorado and available from the web

(http://tornado.atmos.colostate.edu/togadata/ifa_data.html: Ciesielski et al. 2003). The data consists of surface values, and values from $1000 \mathrm{hPa}$ to $25 \mathrm{hPa}$ level with a vertical resolution of $25 \mathrm{hPa}$. Soundings are given every 12 hours.

Fig. 1 shows the vertical profiles of the moist static energy computed by the two definitions (12) and (3) of the enthalpy for the Jordan mean sounding. By adopting the exact expression (12), the moist static energy increases by $15 \mathrm{~K}$ at the surface compared to approximate expression (3). However, we also note that the local absolute value of moist static energy does not play a role in the budget, for the same reason as the constant, $h_{0 l}-h_{0 d}$, can be arbitrary chosen as discussed in the last section. (Note that moist static energy also introduces an arbitrary constant offset for the potential energy, which is typically chosen to be zero so that geopotential height and geometric height above the geoid coincide near the surface.)

In seeking more physical impacts, we now turn to examine the change of the lifting-parcel buoyancy by adopting the exact definition for the moist static energy. Recall that the parcel buoyancy is defined by a difference of the virtual temperature between the parcel and the environment ( $c f$., Roff and Yano 2002). Here, in computing the lifting-parcel buoyancy, the moist static energy is used as a conserved variable for the lifting parcel. The parcel is lifted from the $950 \mathrm{hPa}$ level, as an approximate height for the top of the well-mixed boundary layer. Below the saturation level, the specific vapour value, $q_{v}$, is also conserved, thus the parcel temperature is evaluated in a straightforward manner. Above the saturation level, we set the specific vapour to the saturated value, thus $q_{v}=q_{v}^{*}(T)$, and also assume that the total water, $q_{t}$, is conserved. As a result, the specific liquid water is $q_{l}=q_{t}-q_{v}^{*}(T)$. Under these constraints, parcel temperature at a given level is calculated by a Newton-Raphson method assuming the conservation of the moist static energy.

The resulting buoyancy based on this computation leads to the reversible buoyancy, as no water falls out from the parcel. The alternative definition, called "pseudo-adiabatic" buoyancy, is obtained by setting $q_{l}=0$ in the final result.

The obtained parcel buoyancy under these two definitions for the buoyancy (reversible and pseudo-adiabatic) and the two definitions for the moist static energy are shown in Fig. 2 for the Jordan sounding. The pseudo-adiabatic buoyancy decreases by $1 \mathrm{~K}$ when adopting the exact definition (12), and the reversible buoyancy decreases by a similar but lesser extent. At face value the change in parcel buoyancy is not as dramatic as the change in the absolute value of the moist static energy. However, the relative change can be very large, and for the reversible case we find altitudes where the sign of the buoyancy changes between the two definitions.

The vertical integral of the positive parcel buoyancy under these two definitions leads to reversible and pseudo-adiabatic CAPE convective available potential energy). These two CAPE time series are plotted for the first 30 days of the TOGA-COARE IOP over IFA in Fig. 3(a) and (b) along with the precipitation rate in (c). These time series, again, confirm the conclusion from Fig. 2: though the change of CAPE values by adopting the exact definition (12) is clearly not negligible, it is not dramatic. As discussed for example in Yano et al. (2013), CAPE is useful mainly as a qualitative measure of convective instability. Especially, the value of convective buoyancy is substantially reduced from that of a simple lifting-parcel buoyancy by an entrainment process as suggested for example by Fig. 2 of Yano (2003). Since the uncertainties associated with the convective entrainment (cf., de Rooy et al. 2013) is so large that a difference found in Fig. 3 appears less significant.

However, when using CAPE in a quantitative fashion, the adoption of the exact definition (12) seems imperative. Figure 4 shows scatter plots of two types of CAPE for the whole TOGA-COARE period calculated between the approximate expression (Eq. 3: horizontal axis) and the exact expression (Eq. 12: vertical axis). It is seen that the exact expression of the reversible CAPE consistently gives a value $50 \mathrm{~J} / \mathrm{kg}$ larger than the approximated expression below $250 \mathrm{~J} / \mathrm{kg}$ (a). Above this value, the approximated expression tends This article is protected by copyright. All rights reserved. 
to lead to an underestimation, though such cases are rare. For the pseudo-adiabatic CAPE (b), the approximation (3) consistently overestimates the value by $15 \%$ compared to the exact value. The maximum difference reaches $200 \mathrm{~J} / \mathrm{kg}$ for large CAPE values.

\section{Discussions and Conclusions}

Exact definitions for the atmospheric thermodynamic variables are known in the literature. However, in practical data analysis, these definitions are rarely used. Instead, standard approximations are adopted. Then what kind of consequences would such approximations

have? This paper has examined this question by taking the moist static energy as an example.

At first sight, the exact definition (7) for the moist static energy appears not as physically intuitive as the standard approximate definition (3). Here, we have shown that a re-writing of the definition (12) makes it more physically intuitive. An important corollary this re-writing is the crucial importance of taking into account the temperature dependence in the latent heat.

As emphasized by Marquet (2015), when absolute values of thermodynamic variables are concerned, the reference constant values must also be specified. However, in practical applications, only the relative values across phase transitions (latent heats) are of inlportance. We have explicitly shown that the conservation law for the moist static energy can consistently be defined without defining he absolute values of the enthalpy constants.

Practical consequences of using the exact definition or the standard approximations are examined by computing both the liftingparcel buoyancy value as well as CAPE from tropical data sets based on both of them. We show that the modifications are relatively minor when only qualitative properties of the parcel buoyancy or CAPE are concerned, especially in context of convection parameterization ( $c f$., Yano et al. 2013). For example, the actual value of CAPE for a real parcel would likely be more strongly affected - by an entrainment rate, when the change of buoyancy by entrainment is also taken into account (cf., Fig. 2, Yano 2003). The latter parameter is vastly uncertain (cf., de Rooy et al. 2013). Nevertheless, the two definitions give quantitatively very different values, with approximate equations typically overestimating the reversible CAPE by about $50 \mathrm{~J} / \mathrm{kg}$, and the pseudo-adiabatic CAPE by up to the a $200 \mathrm{~J} / \mathrm{kg}$.

Support of CNRS PICS as well as the University of Reading has enabled this collaboration. 0 Appendix A: Liquid-Water Enthalpy and Reference Constants

The present paper emphasizes that the choice of undefined constants in thermodynamics formulas has no practical consequence: this point is made in Sec. 3. On the other hand, Pauluis et al. (2010) point out a difference of the mean meridional circulations defined in isentropic coordinates under different definitions of entropy: moist and liquid-water.

In this respect, the enthalpy considered in the main text corresponds to the "moist" definition. Alternative definition ("liquid water") is obtained by removing $h_{l}$ from Eq. (4) by Eq. (6) instead of $h_{v}$ to find an alternative form of the expression for specific enthalpy:

$$
\tilde{h}_{w}=c_{p} T-q_{l} L_{0}+h_{d 0}+q_{t}\left(h_{v 0}-h_{d 0}\right)
$$

Here, the subscript $w$ is added to emphasize the "liquid water" definition. An argument parallel to the one made in the main text says, again, that the constants, $h_{d 0}$ and $h_{v 0}$, can be chosen arbitrary by maintaining the consistency with the conservation law of $\tilde{h}_{w}$. 
Thus, by setting $h_{d 0}=h_{l 0}=0$ in Eq. (12), and $h_{d 0}=h_{v 0}=0$ in Eq. (A.1), we obtain two different expressions for the enthalpy:

$$
\begin{gathered}
\tilde{h}=c_{p} T+q_{v} L_{0}, \\
\tilde{h}_{w}=c_{p} T-q_{l} L_{0} .
\end{gathered}
$$

Clearly, these two definitions lead to two different numerical values for enthalpy, as emphasized by Pauluis et al. (2010) in the case of entropy. However, this does not contradict the fact that the enthalpy under both definitions can be used to maintain the consistency with ermodynamic conservation law.

To elucidate this point, we can take the difference of the two definitions:

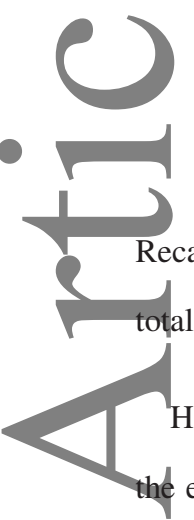

$$
\tilde{h}-\tilde{h}_{w}=q_{t} L_{0}
$$

Recall that the conservation of enthalpy is satisfied only under a conservation of total water, as suggested by Eq. (17). So long as the oțal water is conserved, the above difference, $q_{t} L_{0}$, only contributes a fixed constant.

However, when the condensed water is lost from an air parcel, say, due to the precipitation, the issue becomes more involved. Now, enthalpy is lost by a rate, $h_{l}-h_{d}$ and $h_{v}-h_{d}$, respectively, under the two definitions. From that point, the difference, $q_{t} L_{0}$, no longer remains constant, and as a result, the two definitions of enthalpy will show different spatio-temporal distributions. By applying the same analysis to entropy, Pauluis et al. (2010) show that the isentropic-coordinate based mean circulation analysis leads to different results depending on the choice of the definition of entropy. However, to interpret such circulations in a Lagrangian sense we implicitly (3)

assume some underlying conservation of entropy or moist static energy. As indicated above, this requires Lagrangian conservation of total water content. When this is not the case, the variance in entropy or moist static energy will be due to the varying composition and the subsequent effects of defining the arbitrarily-related zero-point energies of the dry air and water components, as explained in

\section{Sec. 3.
Appendix B: Contribution of Ice to the Moist Static Energy}

The contribution of ice is obtained by adding the ice enthalpy, $h_{i}$, to the formulas presented in the main text. Here, similar to Eq. (6), we find

$$
h_{i}=h_{l}-L_{i}
$$

where $L_{i}$ is the latent heat by ice fusion. As a result, in place of Eq. (7), we obtain

$$
\tilde{h}=\tilde{c}_{p} T+q_{v} L-q_{i} L_{i}
$$

with the definition of $\tilde{c}_{p}$ remaining the same but the contribution of the specific ice, $q_{i}$, included to in the definition of the total specific water, $q_{t}$. Here, the arbitrary constants are set zero for clarity. This expression further reduces to

$$
\tilde{h}=c_{p d} T+q_{v} L-q_{i} L_{i}
$$

under the standard approximation.

This article is protected by copyright. All rights reserved. 
We also note, similar to Eq. (10),

$$
L_{i}=L_{0 i}-\left(c_{i}-c_{p v}\right) T
$$

with $L_{0 i}=3.0 \times 10^{6} \mathrm{~K} / \mathrm{kg}$. Here, by considering a relatively strong temperature dependence of the ice heat capacity, $c_{i}$, we adopt $-20^{\circ} \mathrm{C}$ as a reference temperature in applying Eq. (B.4) in computations below (i.e., $c_{i}=2106 \mathrm{~J} / \mathrm{kg} / \mathrm{K}$ ). Substitution of Eq. (B.4) into Eq. (B.2) leads to the final expression:

$$
\tilde{h}=c_{p} T+q_{v} L_{0}-q_{i} L_{0 i}
$$

Aere, the definition of $c_{p}$ remains the same as in the main text, but the specific ice, $q_{i}$, included to the total water, $q_{t}$.

By introducing these modified definitions, Eqs. (B.3) and (B.5), of the enthalpy into the definition (2) of the moist static energy, we can investigate the change of the reversible parcel buoyancy by adding the ice fusion effect. It is well known that when all the water is frozen above the freezing level, CAPE is increased homogeneously by $1000 \mathrm{~J} / \mathrm{kg}$ approximately in tropical data analysis as shown by egg.,

Roff and Yano (2002). In reality, a substantial fraction of water remains supercooled liquid water below the freezing point. Here, we assume that the fraction of ice among the total condensate linearly increases from zero to unity from $T_{w}=-5^{\circ} \mathrm{C}$ to $T_{i}=-20^{\circ} \mathrm{C}$. We also linearly interpolate the saturated specific water-vapour from $T_{w}$ to $T_{i}$ from the liquid value to the ice value by following rabowski (1998).

The obtained modifications of the reversible buoyancy profile from Fig. 2 are shown in Fig. 5 both with the approximation (Eq. B.3: long dash) and the exact formula (Eq. B.5: solid). Note that the difference of the two curves essentially comes from the errors due to an assumption of a constant ice-fusion heating under Eq. (B.3).

\section{Appendix C: Temperature Dependence of the Liquid Water}

1 As remarked in the main text, the heat capacity of the liquid water is fairly constant down to $-20{ }^{\circ} \mathrm{C}$. Below this temperature, however, its temperature dependence becomes important, as shown by e.g., Fig. 6 of Murphy and Koop (2005). This appendix examines this effect.

Forthis purpose, we idealize the optimal curve shown in Fig. 6 of Murphy and Koop (2005) for the heat capacity of the liquid water yy a curve shown in Fig. 6 (a). Here, a further decrease of the heat capacity below $210 \mathrm{~K}$ is not considered, because that is beyond considered data range. The unit is taken to be $\mathrm{J} / \mathrm{K} / \mathrm{mol}$ in order to facilitate a direct comparison with Fig. 6 of Murphy and Koop (2005), but it can easily be converted into the unit of $\mathrm{J} / \mathrm{K} / \mathrm{kg}$ by dividing it by the molar mass of the water, $18.015 \times 10^{-3} \mathrm{~kg} / \mathrm{mol}$. When the temperature dependence is considered for the heat capacity, the definition of the liquid-water enthalpy must be changed rom

$$
\begin{gathered}
h_{l}=c_{l 0} T \\
h_{l}=h_{0 l}+\int_{0}^{T} c_{l} d T
\end{gathered}
$$

In Eq. (C.1) the subscript 0 is added in defining of the heat capacity in order to explicitly indicate that the heat capacity is constant.

The temperature-dependent heat capacity, $c_{l}$, in Eq. (C.2) may be separated into the two parts:

$$
c_{l}=c_{l 0}+\Delta c_{l}
$$

where $\Delta c_{l}$ is the change of the liquid-water heat capacity by considering its temperature dependence,.

This article is protected by copyright. All rights reserved. 
*References

[1] Ambaum, M. H. P., 2010: Thermal Physics of the Atmosphere, Wiley-Backwell, Chichester, 239pp.

[2] Asai, T., 1965: A numerical study of the air-mass transformation over the Japan Sea in winter. J. Met. Soc. Japan, 43, 1.-15.

[3] Ciesielski, P. E., R. H. Johnson, P. T. Haertel and J. Wang: 2003: Corrected TOGA COARE sounding humidity data: Impact on diagnosed properties of convection and climate over the warm pool. J. Clim., 16, 2370-2384.

[4] de Rooy, W. C., Bechtold, P., Fröhlich, K., Hohenegger, C., Jonker, H., Mironov, D., Siebesma, A. P., Teixeira, J., Yano, J.-I., 2013: Entrainment and detrainment in cumulus convection: an overview, Quart. J. Roy. Meteor. Soc., 139, 1-19, DOI: 10.1002/qj.1959.

Emanuel, K. A., 1994: Atmospheric Convection, Oxford University Press, 580pp.

[6] Grabowski, W. W., 1988: On the bulk parameterization of snow and its application to the quantitative studies of precipitation growth. PAGEOPH, 127, 79-92.

Grabowski, W. W., 1989: On the influence of small-scale topography on precipitation. Quart. J. Roy. Meteor. Soc., 115, 633-650.

8] Grabowski, W. W., 1998: Towards cloud resolving modelling of large-scale tropical circulations: A simple cloud microphysics parameterization. J. Atmos. Sci., 55, 3283-3298.

Hauf, T., and H. Höller, 1987: Entropy and potential temperature. J. Atmos. Sci., 44, 2887-2901.

[10] Jordan, C. L., 1958: Mean sounding for the West Indies area. jm, 15, 91-97.

[11] Kittel, C., and H Kroemer, 1980: Thermal Physics, W. H Freeman \& Company, New York, 473pp.

] Marphy, D. M., and T. Koop, 2005: Review of the vapour pressures of ice and supercooled water for atmospheric applications. Quart. J. Roy. Meteor. Soc., 131, 1539-1565.

[13̧] Marquet, P., 1993: Exergy in meteorology: Definition and properties of moist available enthalpy. Quart. J. Roy. Meteor. Soc., 119, 567-590.

[14] Marquet, P., 2011: Definition of a moist entropy potential temperature: Application to FIRE-I data flights. Quart. J. Roy. Meteor. Soc., 137, 768-791.

15] Marquet, P., 2015: On the computation of moist-air specific thermal enthalpy. Quart. J. Roy. Meteor. Soc., 141, 67-84.

] Marquet, P., and J. F. Geleyn, 2013: On a general definition of the squared Brunt-Väisälä frequency associated with the specific moist entropy potential temperature. Quart. J. Roy. Meteor. Soc., 139, 85-100.

Ooyama, K. V., 1990: A thermodynamic foundation for modeling the moist atmosphere. J. Atmos. Sci., 47, 2580-2593.

[18] Pauluis, O., A. Czaja, and R. Korty, 2010: The global atmospheric circulation in moist isentropic coordinates. J. Climate, 23, $3077-3093$.

[19] Roff, G. L. and J.-I. Yano, 2002: Convective variability in the CAPE phase space. Quart. J. Roy. Meteor. Soc. 128, 2317-2333.

[20] Ritledge, S. A., and P. V. Hobbs, 1983: The mesoscale and microscale structure of clouds and precipitation in midlatitude cyclones. VIII: A model for the 'seeder-feeder' process in warm-frontal rainbands. J. Atmos. Sci., 40, 1185-1206.

Yano, J.-I., 2003: The cumulus parameterization problem in the context of MJO simulations, Proceedings for the MJO workshop, ECMWF, 2-5 November 2003, 115-127.

Yano, J.-I., M. Bister, Z. Fuchs, L. Gerard, V. Phillips, S. Barkidija, and J. M. Piriou, 2013: Phenomenology of convection-parameterization closure. Atmos. Phys. Chem., 13, 4111-4131.

Yau, M. K., and P. M. Austin, 1979: A model for hydrometer growth and evolution of raindrop size spectra in cumulus cells. J. Atmos. Sci., 36, $655-668$. 


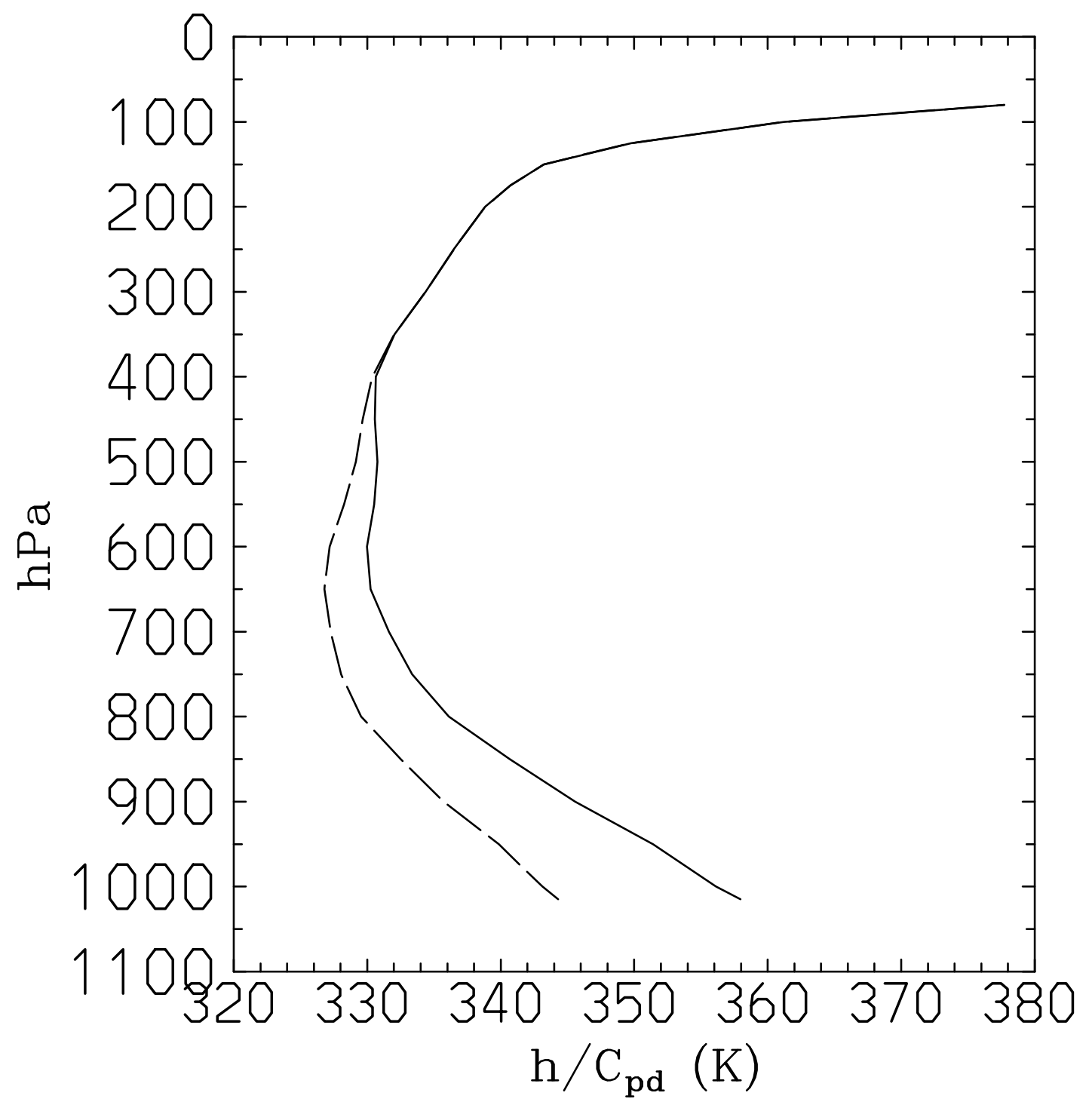

Figure 1. Vertical profiles of the moist static energy obtained under the Jordan sounding: the result based on the exact formula (Eq. 12: solid) and with the standard approximation (Eq. 3: long dash) for the enthalpy. 


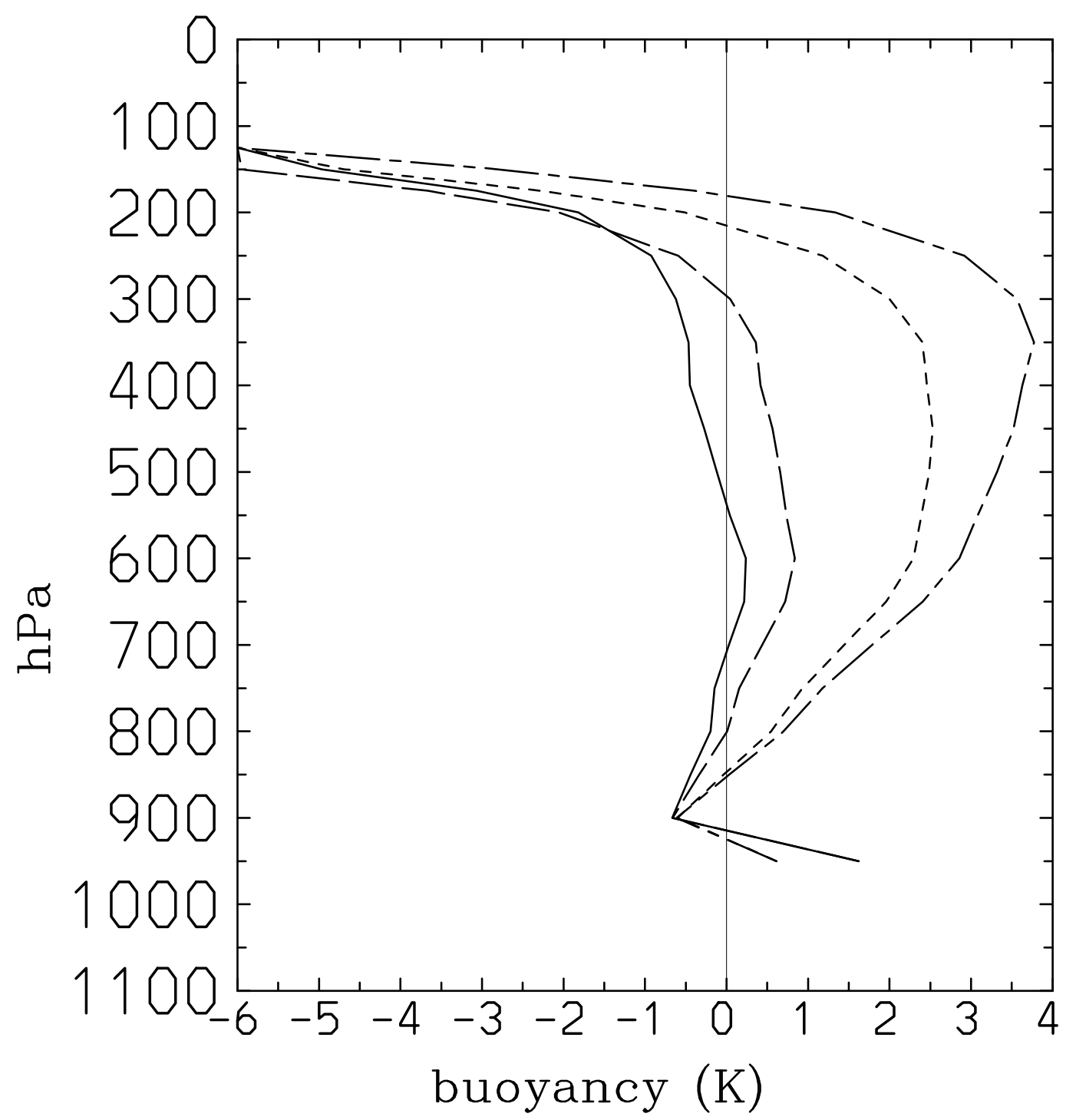

Figure 2. Vertical profiles of the lifting-parcel buoyancy under the Jordan sounding: the solid and long-dash curves (left side) show the reversible case, whereas the shortdash and chain-dash (right side) the pseudo-adiabatic case. The solid and short-dash curves are based on the exact formula (12), whereas the long-dash and chain-dash curves are under the standard approximation (3). 

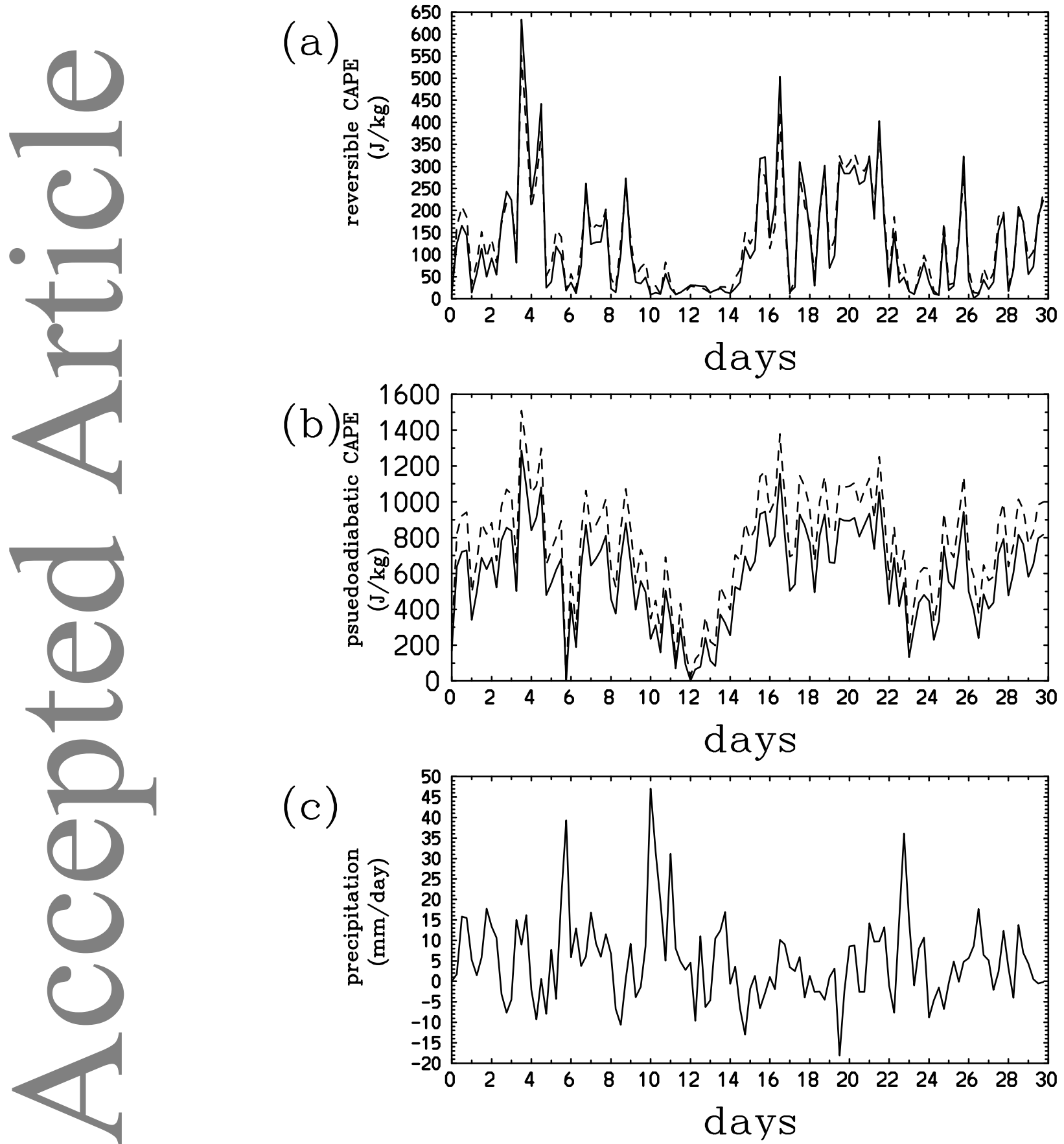

Figure 3. Time series for the first 30 days of the TOGA-COARE period: (a) reversible and (b) pseudo-adiabatic CAPE (J/kg), in which the solid curves show those based on the exact formula (12), the short-dash curves are with the standard approximation (3); (c) precipitation rate (mm/day). Note that the precipitation rate is indirectly estimated from the water-vapour budget, thus it is occasionally negative due to both the observation and diagnosis errors. 

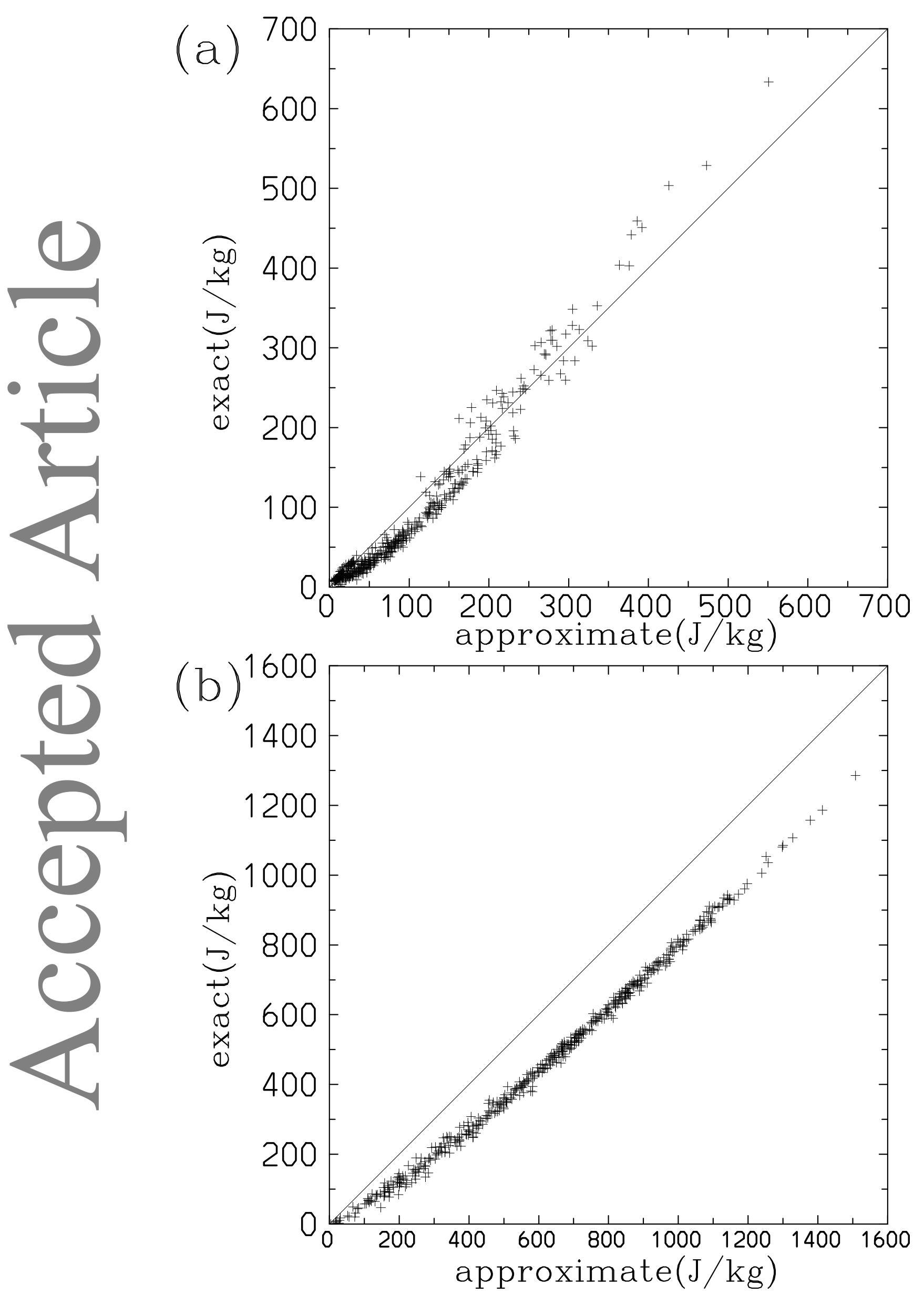

Figure 4. Scatter plots between the exact (vertical axis) and the approximate estimates (horizontal axis) of CAPE based on Eqs. (12) and (3), respectively, for the whole TOGA-COARE period: (a) reversible, (b) pseudo-adiabatic (J/kg).

This article is protected by copyright. All rights reserved. 


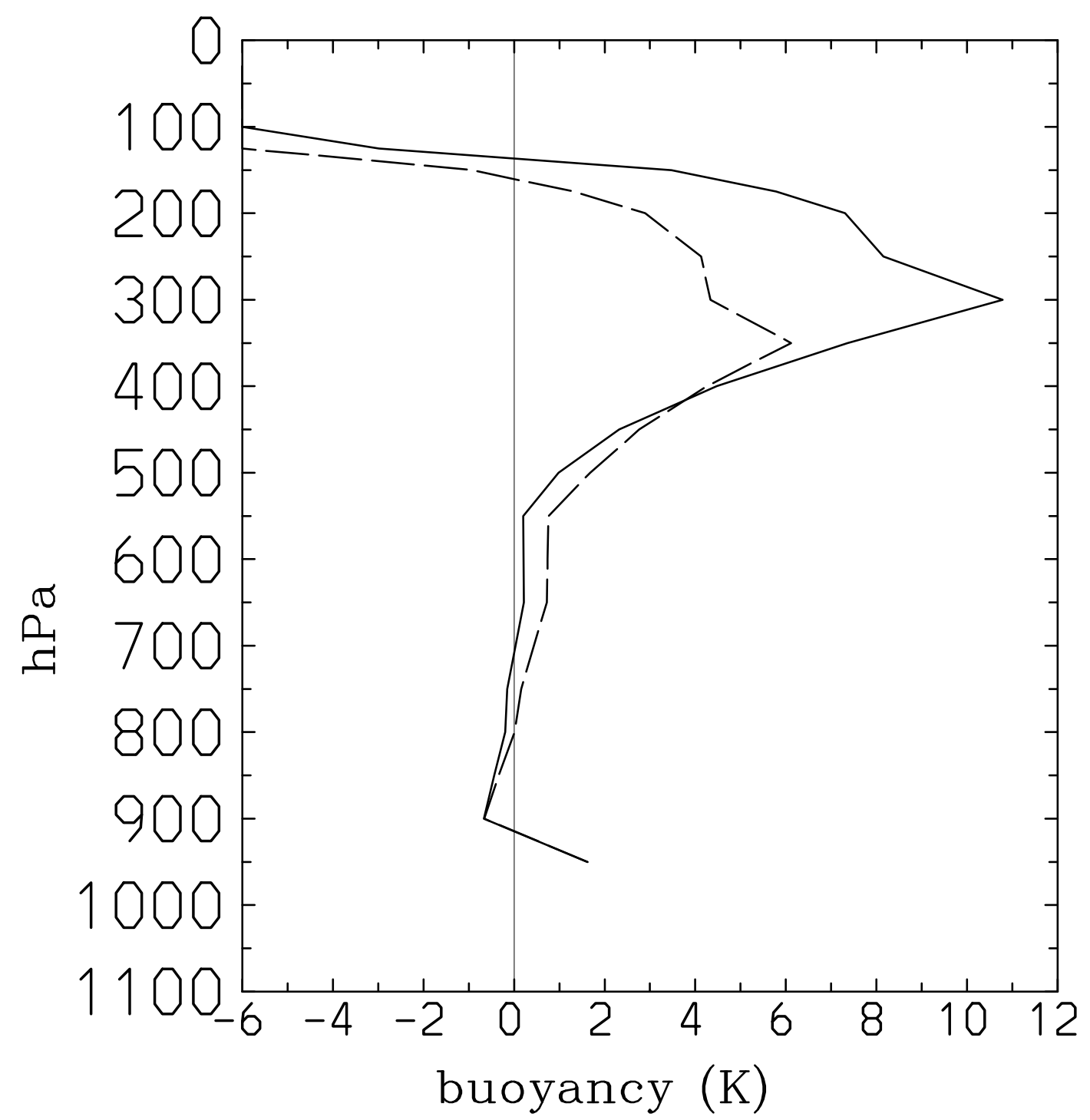

Figure 5. The vertical profiles of the reversible buoyancy, as in Fig. 2, but with the ice fusion effect taken into account: for the exact formula (Eq. B.5: solid), and the approximation (Eq. B.3: long dash). 


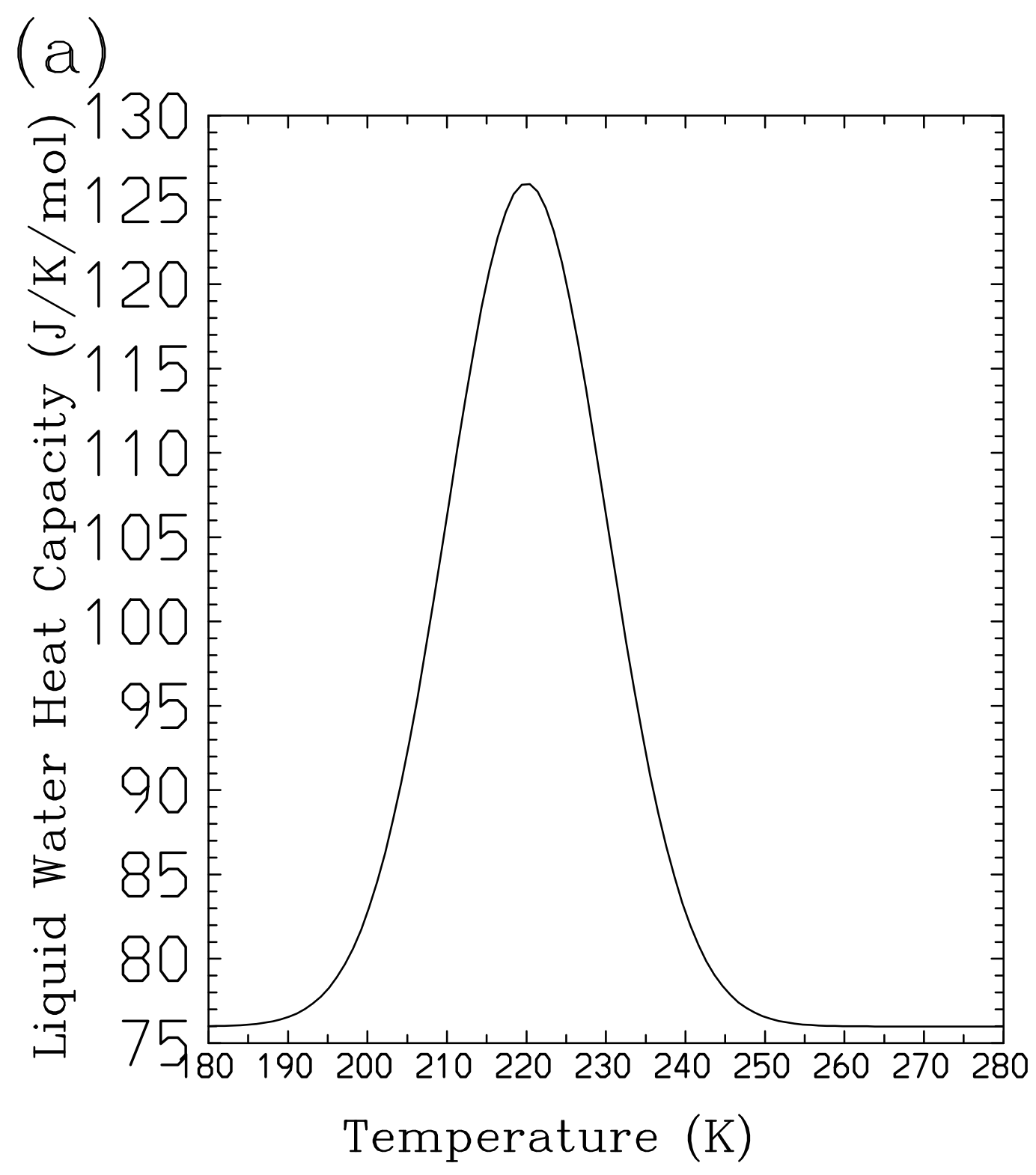


(b)

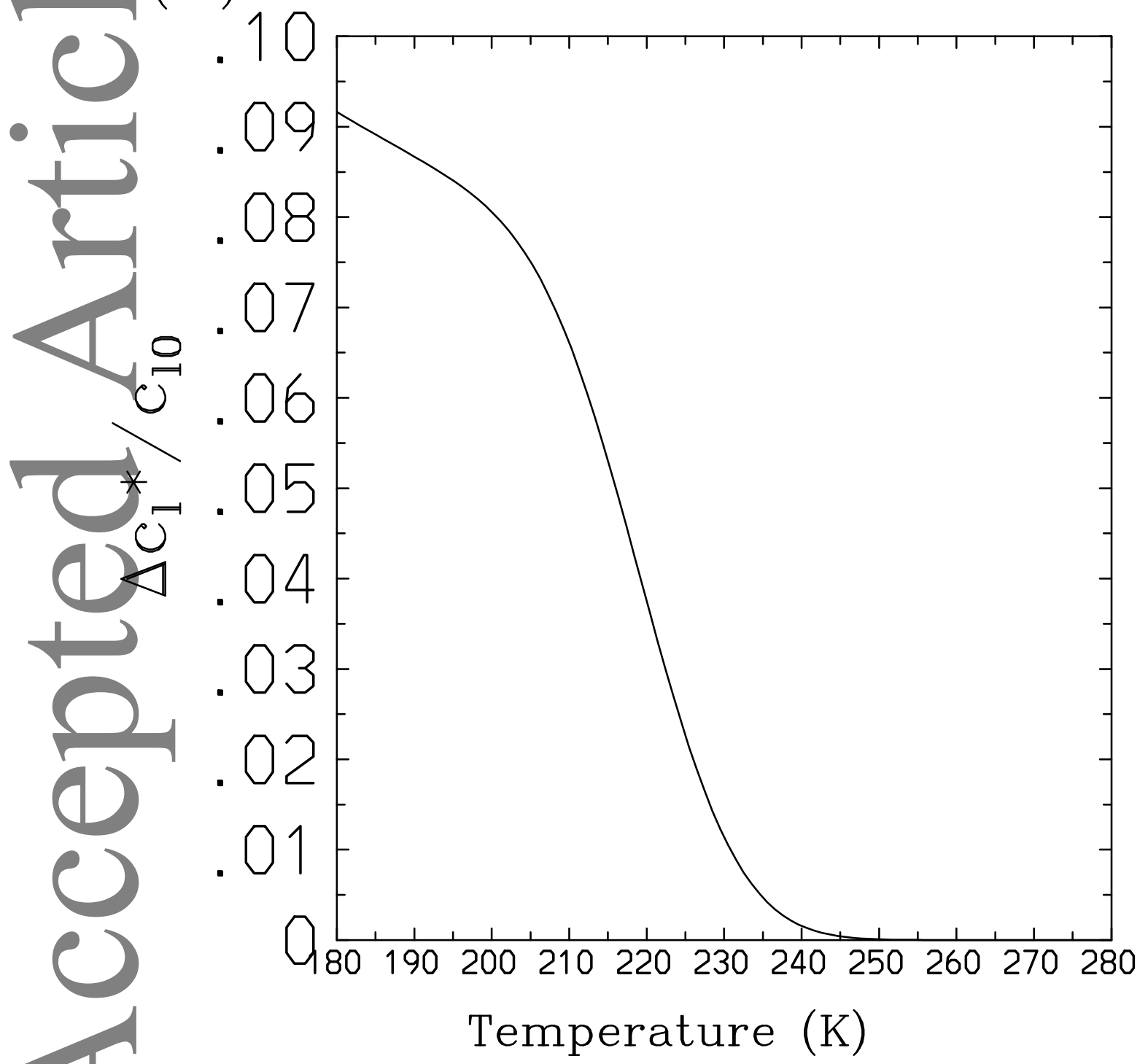

Figure 6. (a) An idealized temperature-dependence of the liquid-water heat capacity considered by following Fig. 6 of Murphy and Koop (2005). (b) A relative change, $c_{l}^{*} / c_{l 0}$, of the effective liquid-water heat capacity, when its temperature dependence is considered as in (a). 


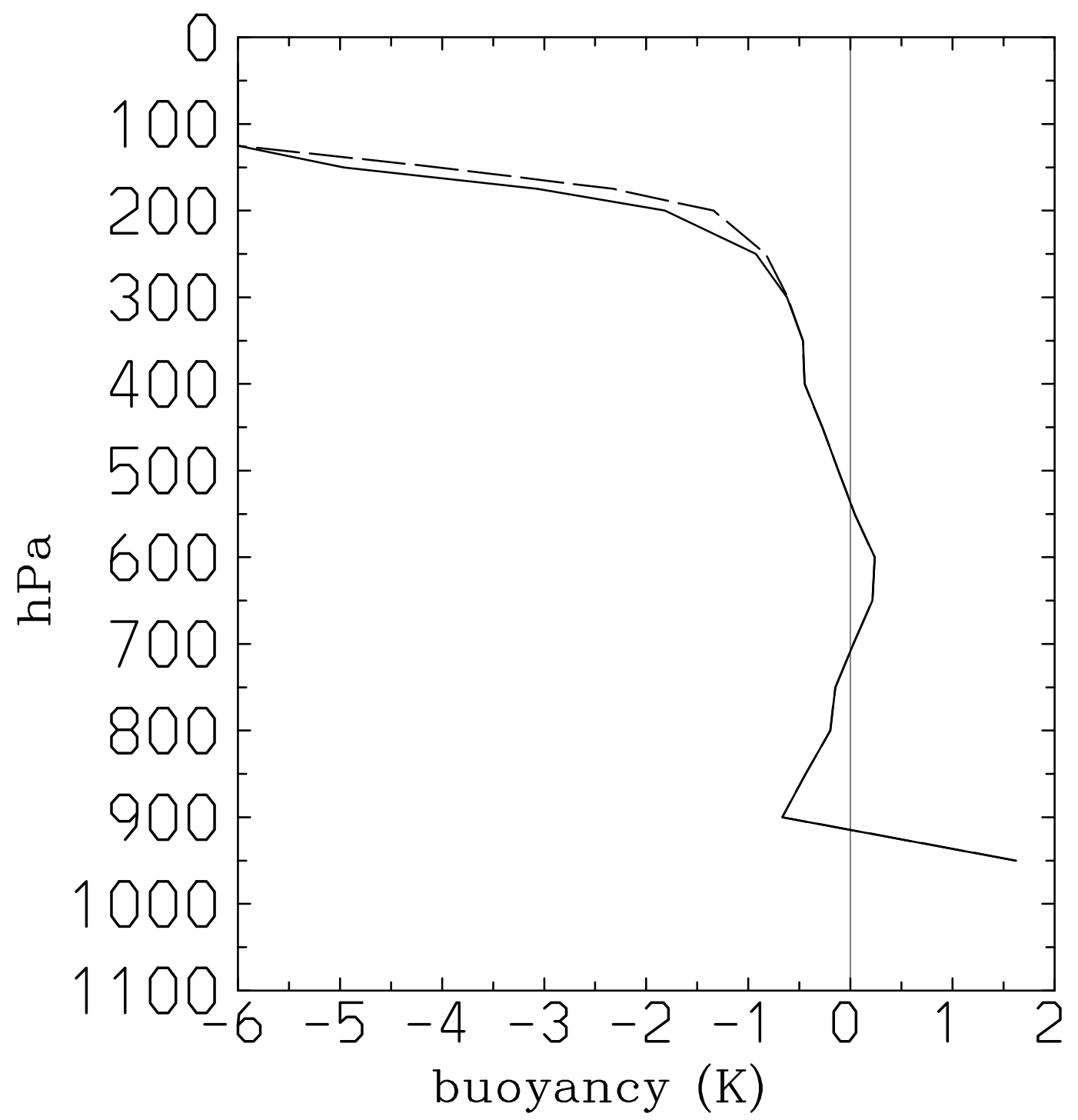

Figure 7. Reversible-buoyancy profile for the Jordan sounding by taking into account of the temperature dependence of the liquid-water heat capacity shown in Fig. 6 (long dash), to be compared against the default case without (solid curve). 